\title{
Goal-concordant care in the ICU: a conceptual framework for future research
}

\author{
Alison E. Turnbull ${ }^{1,2,3^{*}}$ and Christiane S. Hartog ${ }^{4,5}$
}

๑) 2017 The Author(s). This article is an open access publication

The term "goal-concordant care" is increasingly used to describe the objective of interventions aimed at improving clinician-patient communication [1]. The US National Academy of Medicine calls for clinicians to work "with patients and families to ensure that care provided matches closely with each individual's goals" [2]. The German Interdisciplinary Association for Intensive Care and Emergency Care recognizes that patients may reject proposed therapeutic goals if they do not align with patient preferences [3]. Moreover, the first recommendation of the critical care "Choosing Wisely Canada" campaign is to avoid life-supporting interventions "unless they are consistent with the patient's values and realistic goals of care" [4]. Despite increasing interest across medical specialties, no methodology has been validated for measuring goal concordance [5]. Hence, we present a conceptual framework for understanding goal concordance in the adult ICU setting, describe challenges to measuring the incidence of goal-discordant care, and discuss implications for clinical research.

\section{Providing goal-concordant care in the adult ICU}

The adjective "goal-concordant" describes clinical care that helps reach a patient-identified goal, and respects any treatment limitations the patient has placed on clinical care (Fig. 1). Patient goals are individualized, such as living at home, participating in a planned family event, or being surrounded by loved ones at the time of death. Patient goals may thus be distinct from therapeutic goals, such as cure, life prolongation, or palliation [3]. To define potentially achievable goals, the patient or their

\footnotetext{
*Correspondence: turnbull@jhmi.edu

${ }^{2}$ Division of Pulmonary and Critical Care Medicine, School of Medicine, Johns Hopkins University, 1830 E. Monument St, 5th Floor, Baltimore, MD 21205, USA

Full author information is available at the end of the article
}

surrogate(s) must have sufficient prognostic awareness to understand the expected impact of critical illness and treatments on patient survival and subsequent functional outcomes (i.e., physical, cognitive, and mental health status). Because innate patient and family understanding of prognosis is often poor [6], intensivists carefully explain prognosis, and their uncertainty about prognosis, to help a patient or surrogate identify a potentially achievable patient goal [7].

The clinical team also explores whether the patient wishes to place any limitations on the treatments used to achieve their goal. For instance, patients may wish to limit the use of mechanical ventilation and cardiopulmonary resuscitation via do not intubate and do not resuscitate (DNI/R) orders. Given the dynamic nature of critical illness and patient/family understanding of prognosis, regular structured communication with patients/families to reassess patient goals and treatment limitations is essential for goal-concordant care [7].

\section{Measuring the incidence of goal-discordant care for future research}

Estimating the incidence of goal-discordant care requires data on the following throughout a patient's ICU stay: (1) patient goals, (2) treatment limitations if any, (3) treatments received, and (4) judgments regarding whether each received treatment was concordant with the patient's goals and limitations on treatment.

Collecting data on patient goals and treatment limitations poses multiple challenges. First, many medical record systems lack a standardized location to document patient goals. Goals embedded within lengthy progress notes or family meeting notes are difficult to retrieve and not routinely updated. Second, most critically ill patients lack decision-making capacity, leaving family members to represent their interests. However, many family members are unprepared to act as surrogates [8] and are uncertain

\section{勿




\section{Providing Goal-Concordant Care}

Patient or patient surrogate shares

the following with ICU team:

1. The patient's goal - E.g. cure, return to work, live at home, witness a birth or wedding, be comfortable

2. Treatment limitations - Any unacceptable means of achieving their goal e.g. Do not intubate

\section{ICU Clinicians}

1. Determine and explain the patient's diagnosis and prognosis

2. Elicit or clarify patient goals and treatment limitations

3. Appropriately document patient goals and treatment limitations

4. Formulate a treatment plan to maximize probability of achieving patient goals while respecting limitations

5. Routinely check with patient or surrogate for changes to goals and treatment limitations throughout ICU stay
Measuring Goal-Concordance

\section{Measurement Challenges}

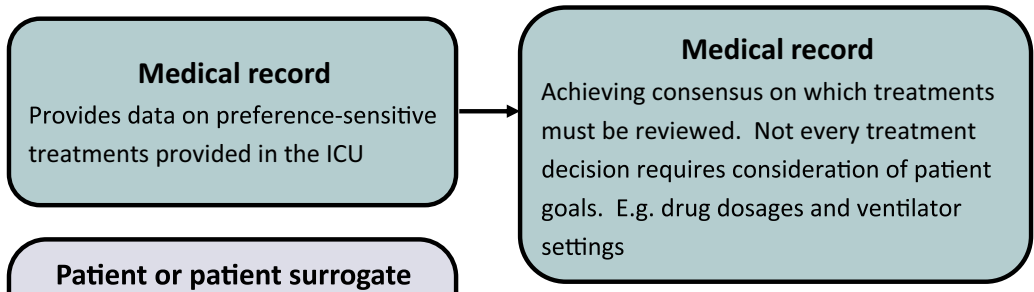

Provides data on patient goals and treatment limitations longitudinally during ICU stay or retrospectively after discharge

Patient or patient surrogate

1. Family unprepared to act as surrogates and uncertain of patient goals and treatment limitations

2. Patients who lack both decision-making capacity and a surrogate

Review data from medical record as well as from the patient or surrogate to determine:

1. Was this patient's goal potentially achievable at the time of the treatment?

2. Will the treatment help achieve the patient's goal and respect the patient's treatment limitations?

Treatments are rated as goalconcordant when the answer to both questions is yes.

Fig. 1 Conceptual framework for clinical research on goal-concordant care in the ICU setting

or incorrect about patient goals and treatment limitations [9]. Missing data is a problem when patients lack both decision-making capacity and a surrogate. Finally, although many countries have established methods to document treatment limitations through advance directives and code status orders, code status extracted from the medical record may not accurately reflect patients' preferred treatment limitations [10, 11]. Hence for research purposes, data on patient goals and treatment limitations should be collected from patients or their surrogates longitudinally during the ICU stay or retrospectively after discharge by trained research personnel using a standardized questionnaire, recognizing that retrospective collection is prone to social desirability bias and introduces a substantial risk for recall bias.

The medical record can provide data on preferencesensitive treatments [7,12] of interest to the research team [11]. Ideally, decisions about which treatments are preference-sensitive for clinical research purposes should be determined with patient-family input [12]. Finally, because these treatments are more common in some
ICUs than in others, we recommend estimating the incidence rate as the number of goal-discordant treatments per 100 preference-sensitive decisions, and reporting confidence intervals around this estimate (ESM Table 1).

Once data on goals, treatment limitations, and treatments are obtained, a method to determine concordance is required. We propose asking two questions: "Was the patient's goal potentially achievable at the time of treatment?" and "Will the treatment help achieve the patient's goal and respect the patient's treatment limitations?" If the answer to both questions is "yes" the treatment is goal-concordant (ESM Table 2). For this approach to be valid, trained clinical reviewers should demonstrate high levels of agreement in response to both questions. Recent research suggests that asking raters how confident they are about the achievability of patient goals could facilitate important sensitivity analyses [13].

\section{Implications for clinical research}

If goal concordance can be measured accurately and reliably, clinical researchers should still hesitate before 
selecting it as a primary outcome. Most interventions target discrete steps in the provision of goal-concordant care (Fig. 1) and in isolation they are unlikely to increase the incidence of goal-concordant treatment. For example, an advance care planning intervention is unlikely to decrease goal-discordant care if ICU clinicians do not routinely elicit and document patient goals. Measuring a more direct effect of the intervention, such as the proportion of patients who report that they have discussed their goals and preferences with their surrogate, is prudent.

Finally, goal concordance is not synonymous with providing treatment perceived as appropriate by clinicians. Clinicians should advocate for the treatment plan they believe is most appropriate given the patient's values [3, 14]. However, patients and their families may set goals that clinicians deride (e.g., patient 5 in ESM Table 2). Using the proposed methodology, treatments that keep patient 5 (ESM Table 2) alive are categorized as goalconcordant despite the fact that some clinicians may consider these treatments to be inappropriate [15].

The proposed process of measuring goal-concordance is valuable because it allows researchers to differentiate between patients whose goals or treatment limitations are unknown, whose goals are unachievable, and whose treatment limitations are ignored. We encourage open discussion, deliberate refinement, and rigorous testing of the proposed methodology for measurement in clinical research.

\section{Electronic supplementary material}

The online version of this article (doi:10.1007/s00134-017-4873-2) contains supplementary material, which is available to authorized users.

\section{Author details \\ 1 Outcomes After Critical IIIness and Surgery (OACIS) Group, Johns Hopkins University, Baltimore, MD, USA. ${ }^{2}$ Division of Pulmonary and Critical Care Medi- cine, School of Medicine, Johns Hopkins University, 1830 E. Monument St, 5th Floor, Baltimore, MD 21205, USA. ${ }^{3}$ Department of Epidemiology, Bloomb- erg School of Public Health, Johns Hopkins University, Baltimore, MD, USA. ${ }^{4}$ Department for Anesthesiology and Intensive Care, Center for Sepsis Control and Care, Jena University Hospital, Erlanger Allee 101, 07743 Jena, Germany. \\ ${ }^{5}$ Patient- and Family-Centered Care, Klinik Bavaria, Kreischa, Germany.}

\section{Acknowledgements}

The authors wish to thank Margaret Hayes, M.D. and Roy Brower, M.D. for their input and critical review.

\section{Compliance with ethical standards}

\section{Conflicts of interest}

On behalf of all authors, the corresponding author states that there is no conflict of interest.

\section{Open Access}

This article is distributed under the terms of the Creative Commons Attribution-NonCommercial 4.0 International License (http://creativecommons.org/ licenses/by-nc/4.0/), which permits any noncommercial use, distribution, and reproduction in any medium, provided you give appropriate credit to the original author(s) and the source, provide a link to the Creative Commons license, and indicate if changes were made.
Received: 23 April 2017 Accepted: 20 June 2017

Published online: 27 June 2017

\section{References}

1. Cooper Z, Koritsanszky LA, Cauley CE et al (2016) Recommendations for best communication practices to facilitate goal-concordant care for seriously ill older patients with emergency surgical conditions. Ann Surg 263:1-6. doi:10.1097/SLA.00000000000001491

2. Dzau VJ, McClellan MB, McGinnis JM et al (2017) Vital directions for health and health care: priorities from a National Academy of Medicine Initiative. JAMA. doi:10.1001/jama.2017.1964

3. Janssens U, Burchardi H, Duttge G et al (2013) Change in therapy target and therapy limitations in intensive care medicine. Position paper of the Ethics Section of the German Interdisciplinary Association for Intensive Care and Emergency Medicine. Anaesthesist 62:47-52. doi:10.1007/ s00101-012-2126-x

4. Critical Care, Choosing Wisely Canada (2017) Critical care: five things clinicians and patients should question. http://www.choosingwiselycanada. org/recommendations/critical-care/. Accessed 26 June 2017

5. Bernacki R, Hutchings M, Vick J et al (2015) Development of the Serious IIIness Care Program: a randomised controlled trial of a palliative care communication intervention. BMJ Open. doi:10.1136/ bmjopen-2015-009032

6. Cox CE, Martinu T, Sathy SJ et al (2009) Expectations and outcomes of prolonged mechanical ventilation. Crit Care Med 37:2888-2894. doi:10.1097/CCM.0b013e3181ab86ed (quiz 2904)

7. Kon AA, Davidson JE, Morrison W et al (2016) Shared decision making in ICUs: an American College of Critical Care Medicine and American Thoracic Society Policy Statement. Crit Care Med 44:188-201. doi:10.1097/ CCM.0000000000001396

8. Majesko A, Hong SY, Weissfeld L, White DB (2012) Identifying family members who may struggle in the role of surrogate decision maker. Crit Care Med 40:2281-2286. doi:10.1097/CCM.0b013e3182533317

9. Torke AM, Sachs GA, Helft PR et al (2014) Scope and outcomes of surrogate decision making among hospitalized older adults. JAMA Intern Med 174:370-377. doi:10.1001/jamainternmed.2013.13315

10. Gehlbach TG, Shinkunas LA, Forman-Hoffman VL et al (2011) Code status orders and goals of care in the medical ICU. Chest 139:802-809. doi:10.1378/chest.10-1798

11. Heyland DK, llan R, Jiang X et al (2016) The prevalence of medical error related to end-of-life communication in Canadian hospitals: results of a multicentre observational study. BMJ Qual Saf 25:671-679. doi:10.1136/ bmjqs-2015-004567

12. Turnbull AE, Sahetya SK, Needham DM (2016) Aligning critical care interventions with patient goals: a modified Delphi study. Heart Lung J Crit Care 45:517-524. doi:10.1016/j.hrtlng.2016.07.011

13. Detsky ME, Harhay MO, Bayard DF et al (2017) Discriminative accuracy of physician and nurse predictions for survival and functional outcomes 6 months after an ICU admission. JAMA. doi:10.1001/jama.2017.4078

14. Bosslet GT, Pope TM, Rubenfeld GD et al (2015) An official ATS/AACN/ ACCP/ESICM/SCCM policy statement: responding to requests for potentially inappropriate treatments in intensive care units. Am J Respir Crit Care Med 191:1318-1330. doi:10.1164/rccm.201505-0924ST

15. Piers RD, Azoulay E, Ricou B et al (2011) Perceptions of appropriateness of care among European and Israeli intensive care unit nurses and physicians. JAMA J Am Med Assoc 306:2694-2703. doi:10.1001/jama.2011.1888 